\title{
Magnetic-field-triggered Remote Drug Release and Analysis of Mechanism
}

\author{
Hongmei Bi, ${ }^{1,2^{*}}$ Zeqin Chen, ${ }^{1}$ Jiaqin Qiu, ${ }^{1}$ and Qianling Chen ${ }^{1}$ \\ ${ }^{1}$ College of Biological and Food Engineering, Guangdong University of Petrochemical Technology, \\ Maoming 525000, China \\ ${ }^{2}$ College of Science, Heilongjiang Bayi Agricultural University, Daqing 163319, China
}

(Received June 14, 2021; accepted July 30, 2021)

Keywords: magnetic trigger, drug release, mechanism, review

A magnetic field is one of the most promising tools for remotely triggered drug release because of its noncontact nature, simplicity, convenience, and excellent maneuverability. A drug delivery system based on magnetic nanoparticles (MNPs) is expected to realize the effective and efficient delivery of drugs with timed quantitatively controlled release in the lesion area. In this review, we give an overview of the recent progress in the fabrication of magnetic drug carriers and magnetically triggered drug release, mainly focusing on the methods of magnetic triggering and the drug release mechanism. In addition, the advantages, limits, and prospects of current triggering methods are also briefly summarized.

\section{Introduction}

The traditional oral administration and injection routes of drugs have many defects such as the easy enzyme degradation of active ingredients in the process of circulation, reduced or no arrival of the drug in the lesion area, the low bioavailability of the drug, and the possibility of a drug overdose and excessive medical treatment. These defects are expected to be solved by the effective delivery of drugs to diseased tissue or to points on a lesion such as tumor areas. ${ }^{(1)}$ Controllable drug delivery and release has major advantages over traditional drug administration in improving the efficiency of drug delivery and reducing systemic toxicity. ${ }^{(2,3)}$ Controlled release can be achieved by the direct control of the drug release process, with the release triggered using smart materials, by changes in the surrounding environment, or by moving or controlling the implanted drug carrier using a noncontact mode to achieve noninvasive, safe, and efficient treatment. ${ }^{(4,5)}$ Among various remote triggering methods, a magnetic trigger has great potential in the development of future targeted and smart drug delivery systems owing to its excellent characteristics such as nontoxicity, high efficiency, noncontact nature, simple equipment, flexible operation, and low side effects. ${ }^{(6)}$ Since the concept of magnetic hyperthermia based on magnetic nanoparticles (MNPs) for cancer treatment was first proposed, research on magnetic drug carriers and magnetically triggered drug release has been widely carried out. ${ }^{(7-9)}$ In this review, the fabrication of magnetic drug carriers and the progress in the

*Corresponding author: e-mail: hongmei_bi@126.com

https://doi.org/10.18494/SAM.2021.3460 
research on magnetically triggered drug release are summarized, mainly focusing on the analysis of the magnetic triggering mode and the drug release mechanism (Fig. 1). The characteristics, advantages, and disadvantages of different release modes are described, and the prospects for the future development of this research field are also discussed.

\section{Drug Release and Mechanism Analysis of Different Trigger Modes}

A magnetic field is the key factor used to trigger the drug release of magnetic drug carriers, and the frequency is the key parameter used to regulate the magnetic field. The different frequencies used, i.e., low frequency, high frequency, radio frequency, and pulsed frequency, are separately discussed in this section.

\subsection{Low-frequency alternating magnetic fields (LF-AMFs)}

The range of $0.01-10 \mathrm{kHz}$ is considered as the low-frequency range for magnetic fields. It is also considered as the safe frequency range of alternating magnetic fields (AMFs) used for magnetically triggered drug release. According to existing reports, $50 \mathrm{~Hz}$ is the minimum frequency of AMFs used in triggering drug release. ${ }^{(10)}$ Bringas et al. ${ }^{(11)}$ loaded methylene blue dye and MNPs into $\mathrm{SiO}_{2}$ nanotubes and then coated the magnetic nanotubes with a temperaturesensitive lipid bilayer [Fig. 2(a)]. An LF-AMF of $50 \mathrm{~Hz}$ was used to promote drug release by changing the permeability of the lipid bilayer through the magnetic thermal effect of the MNPs. Nappini and coworkers ${ }^{(12,13)}$ encapsulated iron cobalt oxide $\left(\mathrm{CoFe}_{2} \mathrm{O}_{4}\right)$ nanoparticles and carboxylfluorescein (CF) into the lumen to construct magnetic drug-loaded liposomes. LFAMFs of $0.2,2.84$, and $5.82 \mathrm{kHz}$ were used to control the CF release. The magnetic thermal effect and magnetic oscillation of the MNPs in the lumen generated some cracks in the lipid

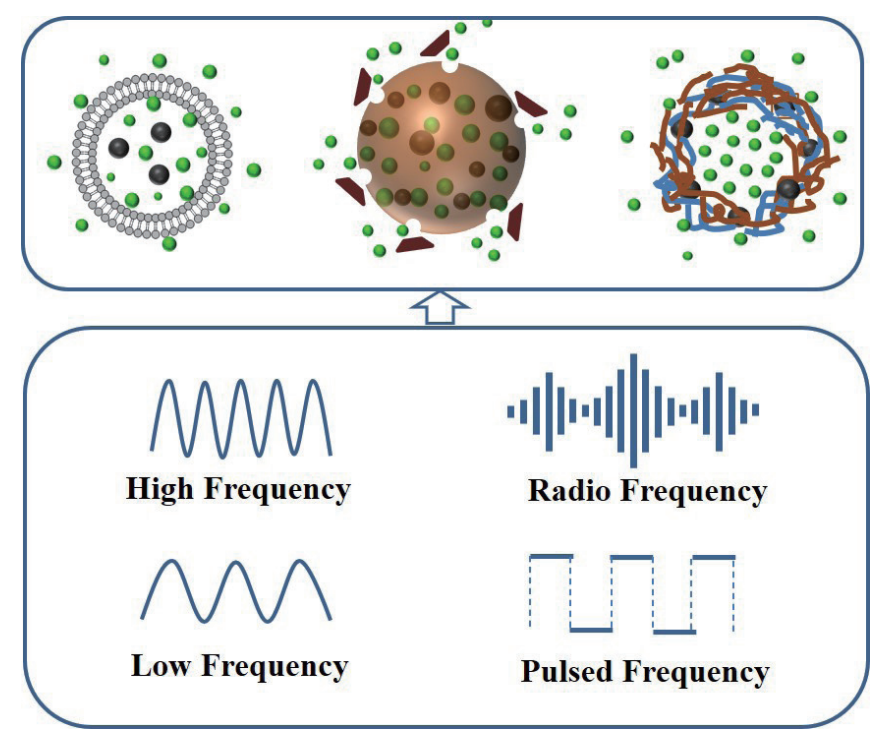

Fig. 1. (Color online) Schematic of magnetically triggered drug release and mechanism. 

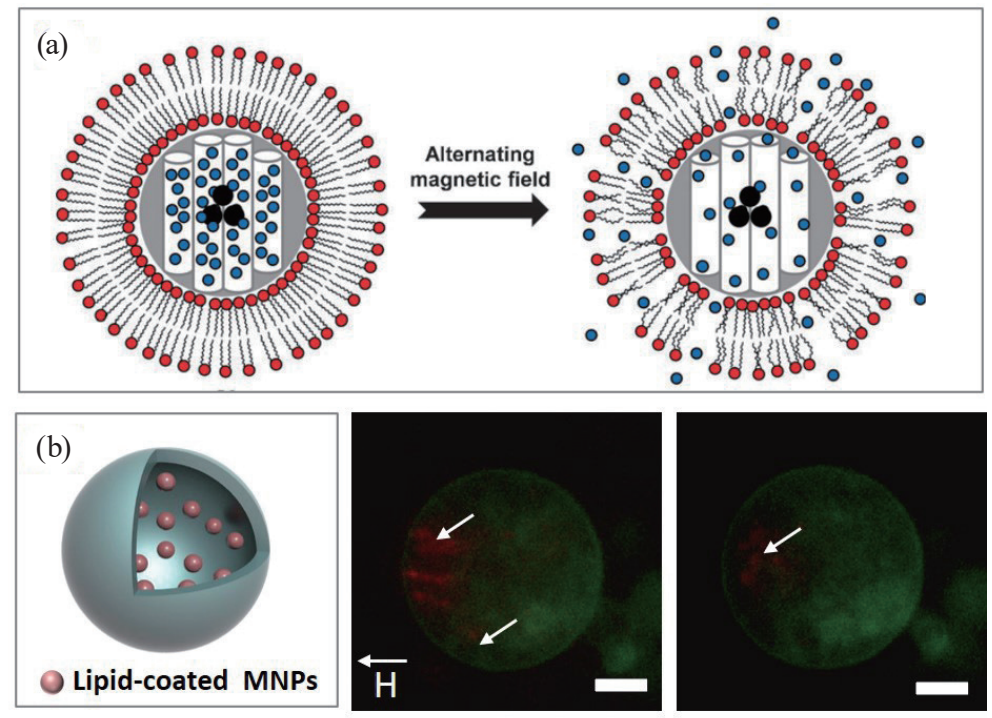

Fig. 2. (Color online) (a) Schematic of magnetic drug carrier structure and drug release. ${ }^{(11)}$ (b) Schematic of magnetic drug carrier structure and magnetic response images. ${ }^{(14)}$

membrane, which affected the permeability of the lipid membrane and led to CF leakage. Our research group ${ }^{(14)}$ coated superparamagnetic $\mathrm{Fe}_{3} \mathrm{O}_{4}$ nanoparticles with a lipid film to increase their monodispersity, colloidal stability, and biocompatibility, and then loaded them into liposomes to fabricate magnetic drug carriers, as shown in Fig. 2(b). No collapse or destruction of the liposomes was observed in the process of CF release by applying AMFs with frequencies of $0.1-5 \mathrm{kHz}$. We considered that the change in the permeability of the lipid membrane was the main reason for the release of the loaded drug. Salvatore et al. ${ }^{(15)}$ used LF-AMFs of 3.22 and $6.22 \mathrm{kHz}$ to study the transport and release of therapeutic oligonucleotides. They designed a multifunctional magnetic nanostructure of lipid/DNA/MNPs, where hydrophobic $\mathrm{Fe}_{3} \mathrm{O}_{4} \mathrm{MNPs}$ were embedded in the middle of the lipid membrane, and core/shell $\mathrm{Au} @ \mathrm{Fe}_{3} \mathrm{O}_{4}$ nanoparticles were functionalized with single-stranded DNA therapy agents and hybridized with cholesterols embedded in the lipid membrane into double-stranded hybrid DNA. The magnetic thermal effect of the MNPs in 3.22 and $6.22 \mathrm{kHz}$ AMFs triggered the unwinding of double-stranded hybrid DNA and released the therapeutic oligonucleotide chains.

Because of their high safety for the human body, LF-AMFs have attracted more attention regarding their potential application for drug release and the orientable transport of biological molecules. Although some achievements have made, there are still obstacles to overcome such as the controllable drug release behavior in the physical medium and the adsorption between the protein and the drug. ${ }^{(16)}$ Most researchers consider that the drug release mechanism triggered by an LF-AMF is based on the permeability change of the carrier shell caused by the magnetic thermal and magnetic oscillation effects, which subsequently promote drug release. ${ }^{(17)}$ In most cases, the role of the magnetic thermal effect is dominant relative to that of the magnetic oscillation effect of MNPs in LF-AMFs. Because the magnetic thermal effect is not strong, especially at very low frequencies, the temperature increase caused by the magnetic thermal 
effect is not significant, making LF-AMFs suitable for a drug delivery system with a lowtemperature requirement. Moreover, the guidance and localization of magnetic nanoclusters and the driving of DNA release controlled by an LF-AMF open new prospects for the development of a nucleic acid-based medicine field.

\subsection{High-frequency alternating magnetic fields (HF-AMFs)}

In general, $10-500 \mathrm{kHz}$ is considered as the high-frequency range in the study of magnetically triggered drug release. An HF-AMF can achieve local heating through hyperthermia and cause drug release without damaging healthy tissues. This frequency range is currently being focused on in the research on magnetically triggered drug release. Guisasola et al ${ }^{(18)}$ reported a nanocomposite drug carrier with a substrate of core/shell $\mathrm{Fe}_{3} \mathrm{O}_{4} @ \mathrm{SiO}_{2}$ nanoparticles and a coated thermosensitive polymer on the surface. They believed that the temperature change induced by the magnetic thermal effect in an AMF of $100 \mathrm{kHz}$ caused the shrinkage of the polymer mesh to promote the rapid release of encapsulated drug molecules and proteins. Fortes Brollo et al. ${ }^{(5)}$ used AMFs of 92, 202, and $282 \mathrm{kHz}$ to remotely trigger the release of the anticancer drug Dox from magnetic liposomes, where MNPs were on the outer layer, in the lumen, and between the lipid bilayers, respectively. They believed that the permeability change of the lipid membrane induced by magnetic heat led to the release of the encapsulated drug regardless of the structure of the magnetic drug carrier. Benyettou et al.$^{(19)}$ prepared a porous magnetic drug carrier using porous carbon as a hard template. Dox was loaded into the pores and the thermosensitive block copolymer Pluronic F108 was coated on the pores. The porous magnetic drug carrier containing Dox was stable at room temperature and physiological $\mathrm{pH}$, but the magnetic thermal effect induced by an HF-AMF of $464 \mathrm{kHz}$ caused the structural shrinkage of Pluronic F108, resulting in the opening of the pores and the sudden release of Dox. Thomas et al. ${ }^{(20)}$ used an AMF of $500 \mathrm{kHz}$ to trigger drug release from a magnetic drug carrier through the removal of the "capping" step, as shown in Fig. 3(a). Firstly, synthesized zinc-doped iron oxide nanocrystals
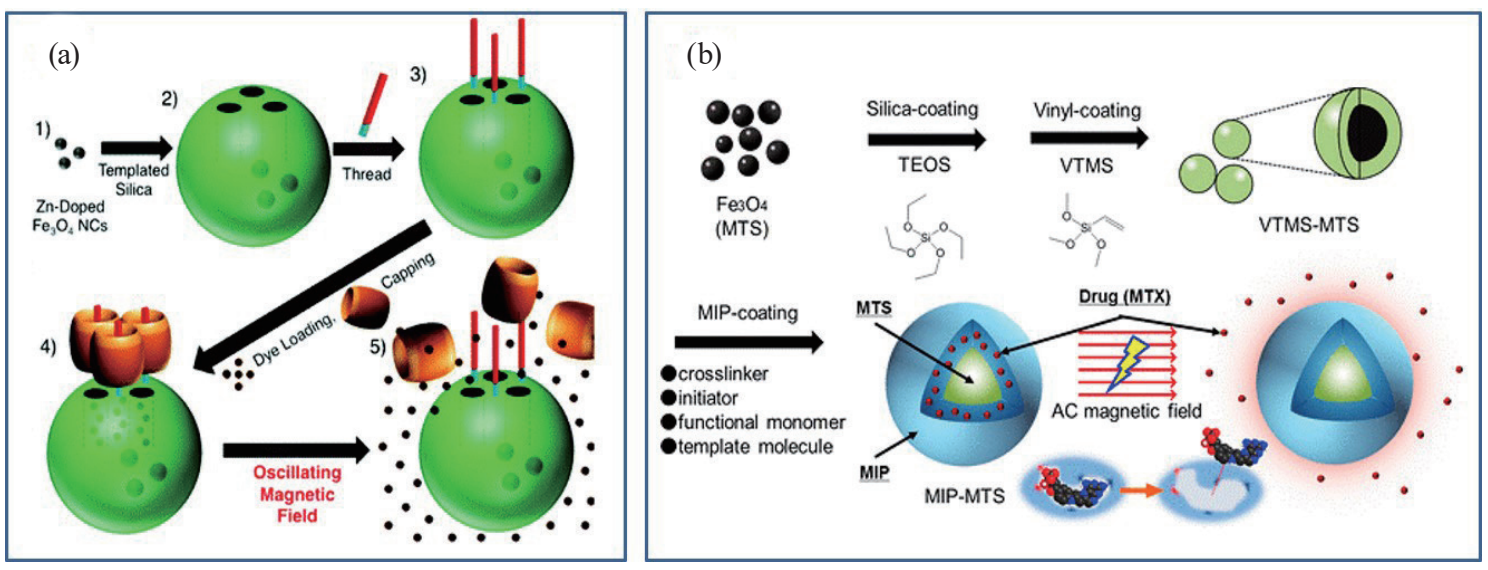

Fig. 3. (Color online) (a) Schematic of porous magnetic drug carrier construction and drug release. ${ }^{(20)}$ (b) Schematic of thermosensitive magnetic drug carrier construction and drug release. ${ }^{(21)}$ 
(ZnNCs) were used as the core, then tetraethyl orthosilicate (TEOS) was wrapped around the $\mathrm{ZnNCs}$ as a catalyst to form magnetic silica particles with a mesoporous structure by hydrolysis, and finally an $N$-(6-N-Aminoethyl) aminomethyltriethoxysilane molecular chain was assembled on the particle surface to increase the magnetic driving force. After Rhodamine B or Dox was loaded into the pores, a molecular chain connected to the surface by cucurbit[6]uril was used as a nanoswitch to cap the pores as shown in Fig. 3(a). The magnetic thermal effect of an AMF of $500 \mathrm{kHz}$ triggered the breakage of the molecular chain, removed the cap, and released the drug inside. The temperature of the solution of this magnetic drug carrier can be up to $52{ }^{\circ} \mathrm{C}$, indicating that the magnetic thermal effect plays a role in the release. Kubo et al. ${ }^{(21)}$ used a molecularly imprinted polymer (MIP) as the carrier of the anticancer drug methotrexate (MTX) to fabricate a magnetic drug carrier, as shown in Fig. 3(b). MIP was coated on vinyl-modified MNPs, and MTX selectively adsorbed on the outer MIP by hydrogen bonding. The generated magnetic heat of the MNPs in an HF-AMF of $600 \mathrm{kHz}$ was able to control the desorption of hydrogen bonds and trigger the release of MTX. This structure is promising for application in multimodal cancer therapy.

HF-AMFs are widely used in magnetically triggered drug release at present. The structure of magnetic drug carriers also varies from magnetic liposomes to porous "capping" materials and polymer materials. ${ }^{(2)}$ Different release mechanisms have also been presented, such as the permeability change of the membrane, shrinkage-induced changes of the drug carrier structure, and intelligent switches. From the perspective of the effect of the magnetic field, the magnetic thermal effect gradually increases with the frequency of the AMF. Although the effect of the magnetic oscillation of MNPs gradually increases with the frequency, it is generally believed that the magnetic thermal effect plays a major role in most drug release systems triggered by an HF-AMF.

\subsection{Radio-frequency (RF) magnetic fields}

RF magnetic fields have also been reported to trigger drug release. In the research on magnetically triggered drug release, the RF range has not been clearly defined, but it usually refers to frequencies above $100 \mathrm{kHz}$. RF overlaps with the high-frequency range and can also be considered as the higher-frequency band of the high-frequency range.

A few research groups have reported drug release studies using RF magnetic fields. Chen et al. ${ }^{(23)}$ reported that 170 and $281 \mathrm{kHz}$ RF magnetic fields triggered the release of Dox from polyethylene glycol (PEG)-stabilized magnetic liposomes. They considered that the magnetic oscillation of MNPs embedded in the lipid membrane destabilized the original equilibrium structure of the lipid membrane and led to the release of Dox, rather than the magnetic thermal effect. Hanus et al. ${ }^{(24)}$ embedded MNPs and drug-loaded liposomes together into an alginate hydrogel. An RF magnetic field of $400 \mathrm{kHz}$ was able to control the temperature of the hydrogel matrix through the magnetic thermal effect of the MNPs. When the temperature was higher than the phase transition temperature of the lipids, the structural change of the lipid membrane led to the release of internal drugs. Kong et al. ${ }^{(25)}$ embedded $\mathrm{Fe}_{3} \mathrm{O}_{4}$ nanoparticles into polystyrene spheres and then polymerized silica in the outer shell to produce a new type of 
hollow magnetic drug carrier by replacing the dissolved polymer material inside the nanoparticles with the anticancer drug camptothecin, as shown in Fig. 4(a). When an RF magnetic field of $100 \mathrm{kHz}$ was intermittently applied in regular intervals, the release of camptothecin synchronized with the application of the magnetic field [Fig. 4(c)]. The drug release mechanism was considered to be mainly based on the temperature increase caused by the magnetic thermal effect rather than the magnetic vibration of MNPs [Fig. 4(b)].

RF can be considered as the higher-frequency band of the HF-AMF. The drug release triggered by an RF magnetic field is similar to that in an HF-AMF. Although the magnetic thermal effect still plays a major role in most drug release systems, the magnetic oscillation of MNPs cannot be ignored in some cases. In particular, at very high frequencies, potential ultrasound will occur and the magnetic oscillation will become strong and play a prominent role in the permeability change or structure collapse of the drug carrier and the drug release.

\subsection{Pulsed magnetic fields}

A pulsed magnetic field was originally discovered and developed by Kapitza during his research on lead-acid batteries. A pulsed magnetic field can provide a strong magnetic field in a short time, which makes it very important in the field of physical science. There have been some reports of its application in remote drug release. Amstad et al. ${ }^{(26)}$ used a $230 \mathrm{kHz}$ pulsed magnetic field to trigger the release of the dye calcein encapsulated in magnetic liposomes. Monodispersed MNPs stabilized with PEG were embedded in the lipid membrane of these magnetic liposomes, which have a phase transition temperature higher than that of the human body [Fig. 5(a)]. After a short period of stimulation with a pulsed magnetic field, the encapsulated
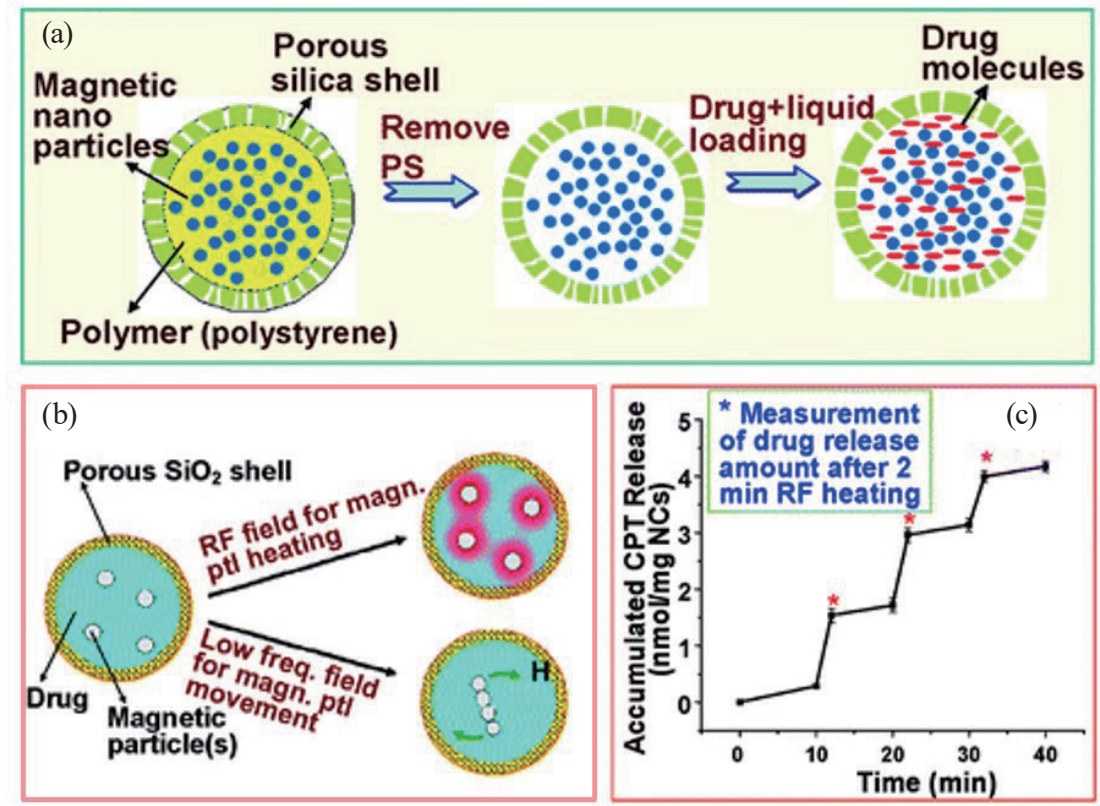

Fig. 4. (Color online) (a) Fabrication of magnetic drug carrier. (b) Schematic of different drug release mechanisms. (c) Drug release curve under RF magnetic field. ${ }^{(24)}$ 

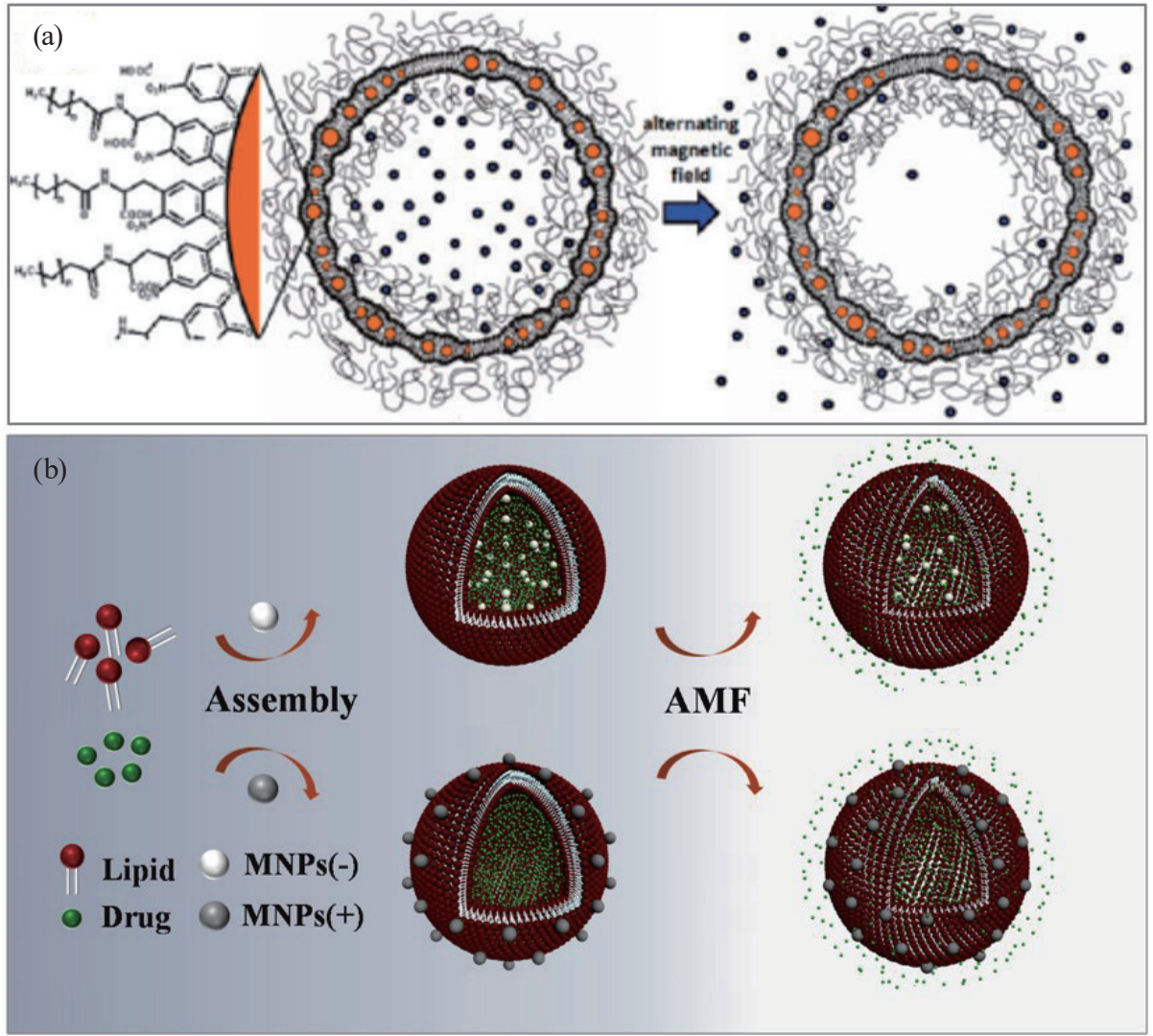

Fig. 5. (Color online) (a) Schematic of liposomes containing MNPs in their bilayer and drug release. ${ }^{(25)}$ (b) Schematic of the structure of two magnetic lipid microcapsules and drug release. ${ }^{(4)}$

drug can be efficiently released without increasing the external temperature to the phase transition temperature. The authors considered that the magnetic oscillation of MNPs under the pulsed magnetic field caused the rupture of the lipid membrane, which resulted in the release of the drug inside. Our group ${ }^{(4)}$ studied the drug release of two different magnetic liposomes using a pulsed magnetic field of $0.5-5 \mathrm{kHz}$ applied at intervals. Two types of magnetic drug-loaded liposome with MNPs in the lumen (internal magnetic type) adsorbed on the surface (external magnetic type) were fabricated [Fig. 5(b)]. A magnetic field triggered the drug release of these two systems, and the release rate and quantity were highly synchronized with the external pulsed magnetic field. From the simulation and experimental results, we concluded that the release mechanism of the internal magnetic drug carrier involved the permeability change of the lipid film caused by the magnetic thermal effect, leading to the release of the drug inside. For an external magnetic drug carrier, the magnetic oscillation effect of MNPs can "tear" the lipid membrane and lead to drug release, and the magnetic thermal effect plays a secondary role.

Podaru et al. ${ }^{(27)}$ adjusted the cholesterol ratio in a lipid and embedded MNPs in a lipid bilayer or encapsuled them in the lumen of liposomes. A short-pulse magnetic field of $30 \mathrm{kHz}$ realized rapid drug release without the local temperature increasing significantly. They considered that the mechanical motion of MNPs under a pulsed magnetic field and the accompanying ultrasonic 
waves destabilized the lipid membrane, causing it to collapse and release the payload in the lumen, whereas the magnetic thermal effect made little contribution to the process. The microrobot-based mechanical motion of MNPs opens up a new direction for the targeted transfer and release of drugs. ${ }^{(28)}$

A pulsed magnetic field can provide a strong magnetic field and a concentrated magnetic thermal effect in a short time, giving it certain advantages in remote-controlled drug release. The magnetic thermal effect, the accompanying magnetic oscillation, and the potential ultrasonic effect in the drug release process give a pulsed magnetic field a wide range of potential applications in controllable drug release, but the fabrication of the drug carrier and the operation of a pulsed magnetic field were relatively complicated. The precision and controllability of a pulsed magnetic field still need to be further explored.

The above four types of magnetic field are the main magnetic fields used in research on magnetic-field-triggered drug release. From the viewpoint of the magnitude, HF-AMFs and RF magnetic fields have a higher frequency than LF-AMFs, the magnetic thermal effect and magnetic oscillation of MNPs in these two magnetic fields are stronger than those in LF-AMFs, and the higher frequency will generate more heat, which is consistent with magneto-thermal theory. Because of the overlap of the frequency range, MNPs exhibit similar behaviors in HFAMFs and RF magnetic fields. According to the mode of action, a pulsed magnetic field is characterized by the intermittent magnetic field, and the frequency and waveform can be adjusted in every pulse. The other three magnetic fields all act in a continuous manner. The contribution of the magnetic thermal effect and magnetic oscillation in drug release and the means of drug release are related to the material composition and the construction of the drug carriers. The four types of magnetic field are briefly summarized in Table 1.

Table 1

Summary of typical microcapsules and effects of different magnetic fields.

\begin{tabular}{|c|c|c|c|c|}
\hline & \multicolumn{4}{|c|}{ Magnetic field classification } \\
\hline & LF-AMFs & HF-AMFs & RF magnetic fields & Pulsed magnetic fields \\
\hline Frequency range & $0.01-10 \mathrm{kHz}$ & $10-500 \mathrm{kHz}$ & $>100 \mathrm{kHz}$ & no defined \\
\hline Effect of MNPs & $\begin{array}{l}\text { Magnetic thermal } \\
\text { effect (dominant) } \\
\text { Magnetic oscillation } \\
\text { (weak, assisting) }\end{array}$ & $\begin{array}{l}\text { Magnetic thermal } \\
\text { effect (dominant) } \\
\text { Magnetic oscillation } \\
\text { (assisting) }\end{array}$ & $\begin{array}{l}\text { Magnetic thermal } \\
\text { effect (dominant) } \\
\text { Magnetic oscillation } \\
\text { (assisting or dominant) }\end{array}$ & $\begin{array}{l}\text { Magnetic thermal } \\
\text { effect (dominant) } \\
\text { Magnetic oscillation } \\
\text { (assisting or dominant) }\end{array}$ \\
\hline $\begin{array}{l}\text { Drug release } \\
\text { mechanism }\end{array}$ & $\begin{array}{l}\text { Permeability change } \\
\quad \text { (liposomes) }\end{array}$ & $\begin{array}{l}\text { Permeability change, } \\
\text { molecular structure/ } \\
\text { orientation change, } \\
\text { removing "capping" } \\
\text { (liposomes, polymer } \\
\text { spheres, inorganic } \\
\text { porous carriers) }\end{array}$ & $\begin{array}{l}\text { Permeability change, } \\
\text { molecular structure/ } \\
\text { orientation change, } \\
\text { removing "capping" } \\
\text { (liposomes, polymer } \\
\text { spheres, inorganic } \\
\text { porous carriers) }\end{array}$ & $\begin{array}{l}\text { Permeability change, } \\
\text { molecular structure/ } \\
\text { orientation change, } \\
\text { structure collapse } \\
\text { (liposomes, polymer } \\
\text { spheres, inorganic } \\
\text { porous carriers) }\end{array}$ \\
\hline $\begin{array}{l}\text { Advantages/ } \\
\text { Disadvantage }\end{array}$ & $\begin{array}{l}\text { Relative safety, } \\
\text { easy fabrication, } \\
\text { low-temperature } \\
\text { requirement }\end{array}$ & $\begin{array}{l}\text { Obvious thermal } \\
\text { effect, controllable } \\
\text { drug release, } \\
\text { wide range of } \\
\text { applications in } \\
\text { relative safety }\end{array}$ & $\begin{array}{l}\text { Obvious thermal } \\
\text { effect, controllable } \\
\text { drug release, } \\
\text { harmful at very high } \\
\text { frequencies }\end{array}$ & $\begin{array}{c}\text { Concentrated } \\
\text { magnetic thermal } \\
\text { effect, controllable } \\
\text { drug release, } \\
\text { complicated operation } \\
\text { low precision }\end{array}$ \\
\hline
\end{tabular}




\section{Conclusions and Prospects}

In this review, we classified and analyzed the progress of remote magnetically triggered drug release and its release mechanism in recent years. Different magnetic trigger methods have their own advantages, limitations, and shortcomings in practical application. LF-AMFs are relatively safe for the human body and the magnetic thermal effect is not obvious. The drug release is mainly achieved by the permeability change of the drug carrier based on the magnetic thermal effect and magnetic oscillation. HF-AMFs can produce a good magnetic thermal effect, and drug release is mostly based on a change in permeability or molecular structure/orientation or the removal of the "capping" step of the drug carrier induced by the magnetic thermal effect. RF magnetic fields behave similarly to HF-AMFs. However, there is a potential for harm at very high frequencies. Pulsed magnetic fields are ideal for controlling drug release, and their application potential is worth further exploration. In addition, we classified the materials used for the fabrication of magnetic drug carriers, and we found that lipids are ideal materials owing to their good biocompatibility and safety. The thickness and strength of a lipid membrane are small, and its permeability and stability can be changed through the magnetic thermal effect and magnetic oscillation of MNPs at different frequencies, leading to the release of encapsulated drugs. Drug carriers constructed from polymer materials have the advantages of stability, high drug loading capacity, and small particle size, but their biosecurity, selectivity, and degradability are not ideal. Generally, drug carrier structures should be destroyed to promote drug release. Inorganic porous materials have a relatively large specific surface and high drug loading capacity. Removing the "capping" step via the magnetic thermal effect is one of the main modes of drug release in inorganic porous magnetic drug carriers. Therefore, the advantages of different materials should be combined to construct new composite materials for the fabrication of magnetic drug carriers in practical applications, and multiple magnetic fields with different frequencies can be used to trigger drug release so as to meet the needs of controllable drug delivery carriers in clinical applications.

\section{Acknowledgments}

This work was supported by the Natural Science Foundation of Guangdong Province (No. 2020A1515010522), Characteristic Innovation Projects of Universities in Guangdong Province (No. 2019KTSCX109), Projects of Talents Recruitment of GDUPT (No. 2019rc114), and Natural Science Foundation of Heilongjiang Province (No. LH2019B012).

\section{References}

1 Z. Ferjaoui, E. Jamal Al Dine, A. Kulmukhamedova, L. Bezdetnaya, C. Soon Chang, R. Schneider, F. Mutelet, D. Mertz, S. Begin-Colin, F. Quilès, E. Gaffet, and H. Alem: ACS Appl. Mater. Inter. 11 (2019) 30610. https:// doi.org/10.1021/acsami.9b10444

2 L. Wang, Y. Chang, Y. Feng, X. Li, Y. Cheng, H. Jian, X. Ma, R. Zheng, X. Wu, K. Xu, and H. Zhang: Nano Lett. 19 (2019) 6800. https://doi.org/10.1021/acs.nanolett.9b01869

3 A. Ahmad, A. Gupta, M. M. Ansari, A. Vyawahare, G. Jayamurugan, and R. Khan: ACS Biomater Sci. Eng. 6 (2020) 1102. https://doi.org/10.1021/acsbiomaterials.9b01947 
4 H. Bi, S. Ma, Q. Li, and X. Han: J. Mater. Chem. B 4 (2016) 3269. https://doi.org/10.1039/C5TB02464A

5 M. E. Fortes Brollo, A. Domínguez-Bajo, A. Tabero, V. Domínguez-Arca, V. Gisbert, G. Prieto, C. Johansson, R. Garcia, A. Villanueva, M. C. Serrano, and M. d. P. Morales: ACS Appl. Mater. Inter. 12 (2020) 4295. https:// doi.org/10.1021/acsami.9b20603

6 A. C. Manjua, V. D. Alves, J. G. Crespo, and C. A. M. Portugal: ACS Appl. Mater. Inter. 11 (2019) 21239. https://doi.org/10.1021/acsami.9b03146

7 H. Bi, D. Fu, L. Wang, and X. Han: ACS Nano 8 (2014) 3961. https://doi.org/10.1021/nn500876z

8 A. I. Martínez-Banderas, A. Aires, M. Quintanilla, J. A. Holguín-Lerma, C. Lozano-Pedraza, F. J. Teran, J. A. Moreno, J. E. Perez, B. S. Ooi, T. Ravasi, J. S. Merzaban, A. L. Cortajarena, and J. Kosel: ACS Appl. Mater. Inter. 11 (2019) 43976. https://doi.org/10.1021/acsami.9b17512

9 S. Fernandes, T. Fernandez, S. Metze, P. Balakrishnan, B. Mai, J. Conteh, C. Mei, A. Turdo, S. Franco, G. Stassi, M. Todaro, and T. Pellegrino: ACS Appl. Mater. Inter. 13 (2021) 15959. https://doi.org/10.1021/ acsami.0c21349

10 A. Joniec, S. Sek, and P. Krysinski: Chem. Eur. J. 22 (2016) 17715. https://doi.org/10.1002/chem.201602809

11 E. Bringas, O. Koysuren, D. V. Quach, M. Mahmoudi, E. Aznar, J. D. Roehling, M. D. Marcos, R. MartinezManez, and P. Stroeve: Chem. Commun. 48 (2012) 5647. https://doi.org/10.1039/c2cc31563g

12 S. Nappini, M. Bonini, F. B. Bombelli, F. Pineider, C. Sangregorio, P. Baglioni, and B. Norden: Soft Matter 7 (2011) 1025. https://doi.org/10.1039/c0sm00789g

13 S. Nappini, M. Bonini, F. Ridi, and P. Baglioni: Soft Matter 7 (2011) 4801. https://doi.org/10.1039/c0sm01264e

14 H. Bi and X. Han: Chem. Phys. Lett. 706 (2018) 455. https://doi.org/10.1016/j.cplett.2018.06.051

15 A. Salvatore, C. Montis, D. Berti, and P. Baglioni: ACS Nano 10 (2016) 7749. https://doi.org/10.1021/ acsnano.6b03194

16 N. Jain, N. Sonker, J. Bajpai, and A. K. Bajpai: ACS. Appli. Bio. Mater. 3 (2020) 3170. https://doi.org/10.1021/ acsabm.0c00178

17 N. Shreyash, M. Sonker, S. Bajpai, and S. Tiwary: ACS Appl. Bio. Mater. 4 (2021) 2307. https://doi.org/10.1021/ acsabm.1c00020

18 E. Guisasola, A. Baeza, M. Talelli, D. Arcos, M. Moros, J. M. de La Fuente, and M. Vallet-Regi: Langmuir 31 (2015) 12777. https://doi.org/10.1021/acs.langmuir.5b03470

19 F. Benyettou, J. A. O. Flores, F. Ravaux, R. Rezgui, M. Jouiad, S. I. Nehme, R. K. Parsapur, J. C. Olsen, P. Selvam, and A. Trabolsi: Chem. Eur. J. 22 (2016) 17018. https://doi.org/10.1002/chem.201602956

20 C. R. Thomas, D. P. Ferris, J. H. Lee, E. Choi, M. H. Cho, E. S. Kim, J. F. Stoddart, J. S. Shin, J. Cheon, and J. I. Zink: J. Am. Chem. Soc. 132 (2010) 10623. https://doi.org/10.1021/ja1022267

21 T. Kubo, K. Tachibana, T. Naito, S. Mukai, K. Akiyoshi, J. Balachandran, and K. Otsuka: ACS Biomater Sci. Eng. 5 (2019) 759. https://doi.org/10.1021/acsbiomaterials.8b01401

22 G. Qian, L. Zhang, X. Li, C. Shuai, and X. Wang: ACS Appl. Bio. Mater. 4 (2021) 5304. https://doi.org/10.1021/ acsabm.1c00422

23 Y. J. Chen, Y. Chen, D. Xiao, A. Bose, R. T. Deng, and G. D. Bothun: Colloid Surf., B 116 (2014) 452.https:// doi.org/10.1016/j.colsurfb.2014.01.022

24 J. Hanus, M. Ullrich, J. Dohnal, M. Singh, and F. Stepanek: Langmuir 29 (2013) 4381. https://doi.org/10.1021/ $\underline{\text { la4000318 }}$

25 S. D. Kong, W. Z. Zhang, J. H. Lee, K. Brammer, R. Lal, M. Karin, and S. H. Jin: Nano Lett. 10 (2010) 5088. https://doi.org/10.1021/nl1033733

26 E. Amstad, J. Kohlbrecher, E. Muller, T. Schweizer, M. Textor, and E. Reimhult: Nano Lett. 11 (2011) 1664. https://doi.org/10.1021/nl2001499

27 G. Podaru, S. Ogden, A. Baxter, T. Shrestha, S. Ren, P. Thapa, R. K. Dani, H. Wang, M. T. Basel, P. Prakash, S. H. Bossmann, and V. Chikan: J. Phys. Chem. B 118 (2014) 11715. https://doi.org/10.1021/jp5022278

28 H. Lee, D. Kim, S. Kwon, and S. Park: ACS Appl. Mater. Inter. 13 (2021) 19633. https://doi.org/10.1021/ acsami. $1 \mathrm{c} 01742$ 


\section{About the Authors}

Hongmei Bi received her B.S. degree from Harbin Normal University, China, in 2001, her M.S. degree from Heilongjiang Bayi Agricultural University, China, in 2005, and her Ph.D. degree from Harbin Institute of Technology, China, in 2016. From 2001 to 2018, she was a lecturer at Heilongjiang Bayi Agricultural University, China. Since 2019, she has been an assistant professor at Guangdong University of Petrochemical Technology. Her research interests are in lipid assembly, drug release, bioengineering, and sensors. (hongmei_bi@126.com)

Zeqin Chen is a junior student at Guangdong University of Petrochemical Technology, China. He assisted in the writing of this review. (1349466080@qq.com)

Jiaqin Qiu is a junior student at Guangdong University of Petrochemical Technology, China. She assisted in the writing of this review. (1790192918@qq.com)

Qianling Chen is a junior student at Guangdong University of Petrochemical Technology, China. She assisted in the writing of this review. (1916266156@qq.com) 
